\title{
HIV integrase inhibitor-based regimen beats market leader
}

Shionogi-ViiV Healthcare has announced positive results from the Phase III SINGLE trial of their investigational HIV integrase inhibitor, dolutegravir, in treatment-naive adults with HIV-1. The dolutegravir-based regimen demonstrated superiority over the most widely prescribed HIV combination therapy, Atripla (Gilead/BMS).

Although there is no cure for HIV infection, progression to symptomatic disease can be halted or delayed in most patients by using a combination of agents targeting different stages of the viral life cycle. The current standard of care is highly active antiretroviral therapy (HAART), which is a triple therapy that consists of a non-nucleoside reverse transcriptase inhibitor (NNRTI), a protease inhibitor or an

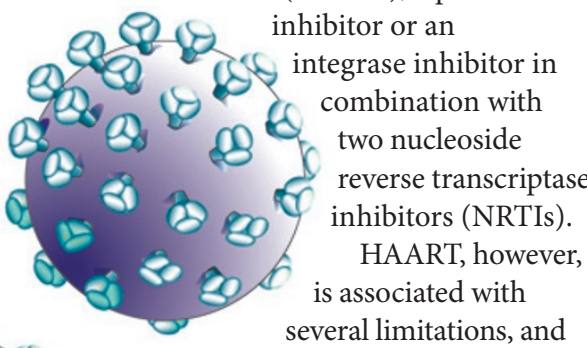

there is continued interest in the development of novel treatments. Emily Erbelding, National Institute of Allergy and Infectious Diseases, Maryland, USA, explains: "Until we can cure HIV, we will always be trying to identify new drug targets so that we can develop more potent drug cocktails or better second line regimens to treat those with viral resistance. Because adherence can be a challenge for some people, we also need to develop very long-acting formulations that are safe and fully suppressive." Andrew Carr, HIV, Immunology and Infectious Diseases Unit, St Vincent's Hospital, Sydney, Australia, adds: "New drugs need to be at least equally potent, but also have additional advantages over existing drugs - lower cost, less toxicity, fewer drug interactions, once daily dosing, and a sufficiently low dose that permits ready co-formulation into single tablet regimens."

HIV integrase inhibitors - which prevent viral DNA from integrating into the genetic material of the host cell, thereby blocking HIV replication - have the potential to address some of the limitations of existing treatments. "Integrase inhibitors are an attractive fourth drug class. They have less CNS toxicity than the NNRTI efavirenz, and generally less gastrointestinal toxicity than protease inhibitors," says Carr. The first drug in this class, raltegravir (Isentress; Merck), received US Food and Drug Administration approval in 2007, and elvitegravir (Gilead) is next in line, closely pursued by dolutegravir.

Results of the Phase III SINGLE trial are encouraging. In the study, 414 patients with HIV received the dolutegravir-based regimen (50 mg dolutegravir plus the NRTIs abacavir and lamivudine) and 419 received Atripla (a single-tablet regimen composed of the NRTIs tenofovir and emtricitabine, and the NNRTI efavirenz) once daily. After 48 weeks, $88 \%$ of dolutegravir-treated patients were virologically suppressed ( $<50$ copies $/ \mathrm{mL}$ ) versus $81 \%$ of patients receiving Atripla. Only $2 \%$ of patients treated with dolutegravir discontinued the study due to adverse events, compared to $10 \%$ of Atripla-treated patients.

"It will be important to see the results of the SINGLE trial in the peer-reviewed literature to fully understand its niche. But from the data we have seen, combination of dolutegravir, abacavir and lamivudine is a once-a-day regimen that is highly effective and well tolerated," says Erbelding.

Dolutegravir may offer advantages over other integrase inhibitors. Indeed, 48-week data from the SPRING-II Phase III trial indicate that once-daily dolutegravir $(50 \mathrm{mg})$ is non-inferior to twice-daily raltegravir $(400 \mathrm{mg}$ ) in treatment-naive adults with HIV-1. "Potential advantages over raltegravir are dolutegravir's once-daily dosing and lower dose, so increasing the potential for co-formulation", notes Carr. "In contrast, elvitegravir has several relative disadvantages: it must be taken with food and to enable once-daily dosing elvitegravir is taken with the cytochrome P450 inhibitor cobicistat. However, elvitegravir has the advantage at present of co-formulation with tenofovir, emtricitabine and cobicistat into a single-tablet regimen; co-formulation of dolutegravir, abacavir and lamivudine is anticipated", he adds.

Sarah Crunkhorn 